\title{
Mathematical analysis of transport systems modeled by the stationary Kolmogorov-Feller equation with a nonlinear drift coefficient
}

\author{
Andrei Firsov ${ }^{1}$, Anton Zhilenkov ${ }^{2}$ \\ Peter the Great St. Petersburg Polytechnic University, Saint Petersburg, Russia \\ ${ }^{2}$ Corresponding author \\ E-mail: ${ }^{1}$ anfirs@yandex.ru, ${ }^{2}$ marine_electronics@corp.smtu.ru \\ Received 12 May 2019; accepted 18 June 2019 \\ DOI https://doi.org/10.21595/vp.2019.20795
}

Check for updates

Copyright (C) 2019 Andrei Firsov, et al. This is an open access article distributed under the Creative Commons Attribution License, which permits unrestricted use, distribution, and reproduction in any medium, provided the original work is properly cited.

\begin{abstract}
The paper proposes the formulation of problems modeled with the stationary Kolmogorov-Feller equations with a nonlinear drift coefficient. Mathematical analysis of the model is given. The basis of the proposed method is the application of the Fourier transform to obtain analytical solutions to the problems under consideration.
\end{abstract}

Keywords: Kolmogorov-Feller equation, nonlinear drift coefficient, constructive method for solving.

\section{Introduction}

Kolmogorov-Feller type equations, which are very popular in solving problems from various fields of natural science (Markov's process theory, control theory, astronomy, physical and chemical processes), are integro-differential equations with (in general) non-linear coefficients. The theory of such equations (from a mathematical point of view) is sufficiently well developed only for the case of coefficients that depend linearly on the independent variable. In the general case, as far as we know, there are few accurate analytical results, and there are practically no strictly grounded constructive methods suitable for applications. In this paper, we propose an analytically justified constructive method for constructing solutions of the corresponding integro-differential equation of Kolmogorov-Feller type for the case of a quadratic dependence of the drift coefficient on the independent variable. The proposed method is very convenient for numerical implementation and, as far as we know, is not found in the literature.

\section{Mathematical model of the problem}

Consider a variant of the Kolmogorov-Feller Eq. (1), which occurs in control theory, communication theory, stellar dynamics. In the literature devoted to analytic constructions of solutions of equations of type Eq. (1), the case of a linear dependence $(\beta=0)$ of the drift coefficient on the coordinate is usually considered; see, for example, [1-7]. In this paper, we will consider $\beta \neq 0$ :

$\frac{d}{d x}\left[\left(\alpha x+\beta x^{2}\right) W(x)\right]+v \int_{-\infty}^{+\infty} p(A) W(x-A) d A-v W(x)=0, \quad-\infty<x<+\infty$,

under natural conditions:

$$
\begin{aligned}
& W(x) \underset{x \rightarrow \pm \infty}{\rightarrow 00}, \quad \int_{-\infty}^{+\infty} W(x) d x=1, \\
& p(A) \underset{|A| \rightarrow \infty}{\longrightarrow} 0, \quad \int_{-\infty}^{+\infty} p(A) d A=1 .
\end{aligned}
$$


Additionally, suppose $p(A)$ - analytical function with $|A|<R$ for $R$ big enough or at least its Fourier transform $\hat{p}(k)=\int_{-\infty}^{+\infty} p(x) e^{i x k} d x$ exists and is an analytical function in a sufficiently large interval:

$\hat{p}(k)=\hat{p}_{0}+\hat{p}_{1} k+\hat{p}_{2} k+\ldots, \quad|k|<k_{0}, \quad k_{0} \gg 1$.

Note that:

$\hat{p}_{s}=\frac{\hat{p}^{(s)}(0)}{s !}=\frac{1}{s !}(i)^{s} \int_{-\infty}^{+\infty} x^{s} p(x) d x$.

In particular, by virtue of Eqs. $(2,3)$ :

$\hat{p}_{0}=\hat{p}(0)=1$.

Also, if $p(x)$ - even function, then $\hat{p}_{2 s-1}=0, s=1,2, \ldots$, and $\hat{p}(k)$ is real.

We now turn in Eq. (1) to the Fourier transform of the function $W(x)$ :

$\widehat{W}(k)=\int_{-\infty}^{+\infty} W(x) e^{i x k} d x$,

$k\left[i \beta \widehat{W}^{\prime \prime}-\alpha \widehat{W^{\prime}}+v\left(\frac{\hat{p}-1}{k}\right) \widehat{W}\right]=0$.

Conditions Eq. (2) at the same time go to:

$\left\{\begin{array}{l}\int_{-\infty}^{+\infty}|\widehat{W}(k)| d k<\infty, \\ \widehat{W}(0)=1 .\end{array}\right.$

Thus, the problem of solving an Eq. (1) with conditions Eq. (2) can be replaced by a solution of an equation:

$i \beta \widehat{W^{\prime \prime}}(k)-\alpha \widehat{W}^{\prime}(k)+v \rho(k) \widehat{W}(k)=0$,

satisfying conditions Eq. (7). Denoted here:

$\rho(k)=\frac{\hat{p}(k)-1}{k}$.

By virtue of Eq. (6), and the conditions imposed on $\hat{p}(k)$, we have:

$\rho(0)=\hat{p}_{1}=\int_{-\infty}^{+\infty} x p(x) d x$.

$\rho(k)=\hat{p}_{1}+\hat{p}_{2} k+\hat{p}_{3} k^{2}+\ldots, \quad|k|<k_{0}$.

Moreover, since $\hat{p}(k) \rightarrow 0,|k| \rightarrow \infty$ (because $\hat{p}$ is a Fourier transform), then:

$\rho(k) \sim \frac{-1}{k}, \quad(|k| \rightarrow \infty)$. 


\section{Mathematical model analysis}

Assume:

$\widehat{W}(k)=\varphi(k) e^{-\int_{0}^{k} \psi(k) d k}$

Notice, that:

$\varphi(0)=\widehat{W}(0)=1$,

$\varphi(-k)=\overline{\varphi(k)}$.

(The last equality is a consequence of the choice of $\psi(k)$, which will be done below - see Eq. (16)). Substituting Eq. (12) into Eq. (8), we get:

$\varphi^{\prime \prime}+\left(-2 \psi+i \frac{\alpha}{\beta}\right) \varphi^{\prime}+\left(\psi^{2}-\psi^{\prime}-i \frac{\alpha}{\beta} \psi-i \frac{\nu}{\beta} \rho\right) \varphi=0$.

Assuming:

$\psi=i \frac{\alpha}{2 \beta}$,

yields the following equation for $\varphi(k)$ :

$\varphi^{\prime \prime}-q(k) \varphi=0$,

where:

$\varphi(k)=\widehat{W}(k) e^{i \frac{\alpha}{2 \beta} k}$,

$q(k)=-\frac{\alpha^{2}}{2 \beta^{2}}+i \frac{v}{\beta} \rho(k)$.

Let us note some elementary properties of $q(k)$.

1) By virtue of Eq. (12):

$q(k)+\frac{\alpha^{2}}{2 \beta^{2}} \sim-i \frac{v}{\beta k}, \quad(|k| \rightarrow \infty)$,

and in particular:

$q(k) \rightarrow-\frac{\alpha^{2}}{2 \beta^{2}}, \quad(|k| \rightarrow \infty)$.

2) By virtue of Eq. (11):

$q(k)=-\frac{\alpha^{2}}{2 \beta^{2}}+i \frac{v}{\beta} \hat{p}_{1}+i \frac{v}{\beta}\left(\hat{p}_{2} k+\hat{p}_{3} k^{2}+\ldots\right), \quad|k|<k_{0}$,

or

$q(k)=q_{0}+q_{1} k+q_{2} k^{2}+\ldots, \quad|k|<k_{0}$, 
where:

$\left\{\begin{array}{l}q_{0}=-\frac{\alpha^{2}}{2 \beta^{2}}+i \frac{v}{\beta} \hat{p}_{1} \\ q_{n}=i \frac{v}{\beta} \hat{p}_{n+1} .\end{array}\right.$

3) Let:

$\delta=\delta(k)+\frac{\alpha^{2}}{2 \beta^{2}}+\frac{v}{\beta} \operatorname{Im} \rho(k)$.

Then:

$q(k)=-\delta(k)+i \frac{v}{\beta} \operatorname{Re} \rho(k)$.

At the same time:

- if $\rho(k)$ is real (which will take place, if, for example, $p(x)$ - is an even function), then:

$\delta=$ const $=\frac{\alpha^{2}}{2 \beta^{2}}>0$.

- if $\operatorname{Im} \rho(k) \neq 0$, then by Eq. (12), for sufficiently large $|k|$ :

$\delta(k)=\frac{\alpha^{2}}{4 \beta^{2}}+\frac{v}{\beta} \operatorname{Im} \rho(k)>0$.

Lemma 1. The branch of $\sqrt{q(k)}$ :

$$
\begin{gathered}
(\sqrt{q(k)})_{1}=\sqrt{|q(k)|}\left\{\frac{1}{2}\left(1+\left(1+\frac{[\operatorname{Re} \rho(k)]^{2} v^{2}}{\delta^{2} \beta^{2}}\right)^{-\frac{1}{2}}\right)\right\}^{1 / 2} \\
-i \sqrt{|q(k)|}\left\{\frac{1}{2}\left(1-\left(1+\frac{[\operatorname{Re} \rho(k)]^{2} v^{2}}{\delta^{2} \beta^{2}}\right)^{-\frac{1}{2}}\right)\right\}^{1 / 2},
\end{gathered}
$$

is twice continuously differentiable by $k \in(0,+\infty)$ and $\operatorname{Re}(\sqrt{q(k)}){ }_{1}>0$ for $k$, which are large enough.

Remarks:

1) $\sqrt{|q(k)|}=\left(\delta^{2}+\frac{v^{2}}{\beta^{2}}[\operatorname{Re} \rho(k)]^{2}\right)^{1 / 4}$.

2) If $\rho(k)$ - is real, then $\operatorname{Re}(\sqrt{q(k)})_{1}>0$ for all $k>0$.

3) By virtue of property Eq. (15), it suffices to construct a solution to Eq. (17) only for $k \geq 0$ [6-11].

\section{Results and conclusions}

An algorithm for the analytical solution of the Kolmogorov-Feller Eq. (1) is constructed and substantiated. This algorithm is as follows. 
1) Enter the function $\varphi(k)=\widehat{W}(k) e^{i \frac{\alpha}{2 \beta} k}$, where $\widehat{W}(k)$ is a Fourier transform of the desired function $W(x)$.

2) For function $\varphi(k)$ the equation holds:

$\varphi^{\prime \prime}(k)-q(k) \varphi(k)=0, \quad k>0$,

under conditions:

$\left\{\begin{array}{l}\varphi(0)=1, \\ \varphi(k) \rightarrow 0, \quad k \rightarrow+\infty,\end{array}\right.$

$q(k)=q_{0}+q_{1} k+q_{2} k^{2}+\ldots, \quad|k|<k_{0}$.

3) The coefficients $q_{j}$ are from the relations:

$$
\left\{\begin{array}{l}
q_{0}=-\frac{\alpha^{2}}{2 \beta^{2}}+i \frac{v}{\beta} \hat{p}_{1}, \\
q_{n}=i \frac{v}{\beta} \hat{p}_{n+1},
\end{array}\right.
$$

where $\hat{p}_{j}$ are from decomposition coefficients of the Fourier transform $\hat{p}(k)=\int_{-\infty}^{+\infty} p(x) e^{i x k} d x$.

4) The sought solution $\varphi(k)$ can be determined from a system of linear algebraic equations, which will be given in further work.

\section{References}

[1] Tikhonov V. I., Mironov M. A. Markov Processes. Soviet Radio, Moscow, 1977, (in Russian).

[2] Tikhonov V. I., Kharisov V. N. Statistic Analysis and Synthesis of Radio Devices and Systems. Radio and Communication, Moscow, 1991, (in Russian).

[3] Aleksandrov V. D. Exact solution of the stationary Kolmogorov-Feller equation. Review of Applied and Industrial Mathematics, Vol. 9, Issue 1, 2002, p. 106, (in Russian).

[4] King I. R. An Introduction to Classical Stellar Dynamics. University of California, Berkeley, 1994.

[5] Artemyev V. M., Ivanovskyi A. V. Discrete Control Systems with a Random Quantization Period. Moscow, Energoatomizdat, 1986, (in Russian).

[6] Maslov V. P. The Kolmogorov-Feller equation and the probabilistic model of quantum mechanics. Journal of Soviet Mathematics, Vol. 23, Issue 5, 1983, p. 2534-2553.

[7] Rudenko O. V., Dubkov A. A., Gurbatov S. N. On exact solutions to the Kolmogorov-Feller equation. Doklady Mathematics, Vol. 94, Issue 1, 2016, p. 476-479.

[8] Slepova L., Zhilenkov A. Approximating the solution of thermal conductivity problem for composite medium. IEEE Conference of Russian Young Researchers in Electrical and Electronic Engineering (EIConRus), 2019.

[9] Dang B., Zhilenkov A. Numerical solutions for nonhomogeneous thermal conductivity problems in composite layered media. IEEE Conference of Russian Young Researchers in Electrical and Electronic Engineering (EIConRus), 2019.

[10] Sokolov S., Zhilenkov A., Chernyi S., Nyrkov A., Mamunts D. Dynamics models of synchronized piecewise linear discrete chaotic systems of high order. Symmetry, Vol. 11, Issue 2, 2019, p. 236-1.

[11] Glebov N., Zhilenkov A., Chernyi S., Sokolov S. Process of the positioning complex modeling objects with elements of intellectual analysis. Procedia Computer Science, Vol. 150, 2019, p. 609-615. 